\title{
Computation of the Reachability Graph of untimed Hybrid Petri nets
}

\author{
Estíbaliz Fraca, Jorge Júlvez, Manuel Silva \\ GISED (Grupo de Ingeniería de Sistemas Discretos) \\ Instituto de Investigación en Ingeniería de Aragón (I3A). \\ Universidad de Zaragoza, Mariano Esquillor s/n, 50018, Zaragoza, Spain. \\ Tel.+34-976762472, Fax +34-976761914, e-mail: \{efraca,julvez,silva\}@unizar.es
}

\begin{abstract}
Untimed hybrid Petri nets are a formalism for the analysis of dynamical systems, which combines discrete and continuous behaviour. The study of its reachability is interesting for analysis purposes, such as the study of behavioural properties. A method to compute its reachability graph and reachability space is proposed here.
\end{abstract}

\section{Introduction}

Petri nets are a well known family of formalisms for the analysis of Discrete Event Systems, which suffer from the state explosion problem. Hybrid Petri nets (HPN) try to overcome this problem by a partial relaxation of the formalism, where some transitions have a continuous behaviour, while some other remain discrete.

The study of its reachability is interesting for analysis pruporses, such as the study of behavioural properties. The set of markings which are reachable from the initial one is denoted reachability space (RS); while the graphical representation which shows how these markings are reached is denoted reachability graph (RG).

\section{Related work}

The reachability graph of untimed HPN was studied in David and Alla (2010). In the next sections, we compare our method with that one.

Moreover, reachability of timed HPN has been studied in some other works, such as Balduzzi et al. (2001), which reachability of First-Order Hybrid Petri Nets is considered.

This work is to provides a characterization of the RS and RG of untimed HPN. They are obtained by means of an algorithm, and illustrated with an example obtained from the literature.

\section{Reachability graph computation}

The RS of HPN is the result of discrete and continuous firings from the initial state $\mathbf{m}_{0}$. And it has characteristics of both.

The RS of discrete PN systems (David and Alla, 2010 ) is a set of disjointed points in the $\mathbb{N}^{|\mathrm{P}|}$ space. In contrast, the RS of continuous PN systems is a convex set in $\mathbb{R}^{\mathbb{P}_{\geq 0}}$ (Júlvez et al., 2003). It means that, if $\mathbf{m}_{1}$ and $\mathbf{m}_{2}$ are reachable, then for any $\alpha \in[0$, $1], \alpha \mathbf{m}_{1}+(1-\alpha) \mathbf{m}_{2}$ is also reachable. Due to the continuous and discrete firings, the RS can be seen as a "set of convex sets".

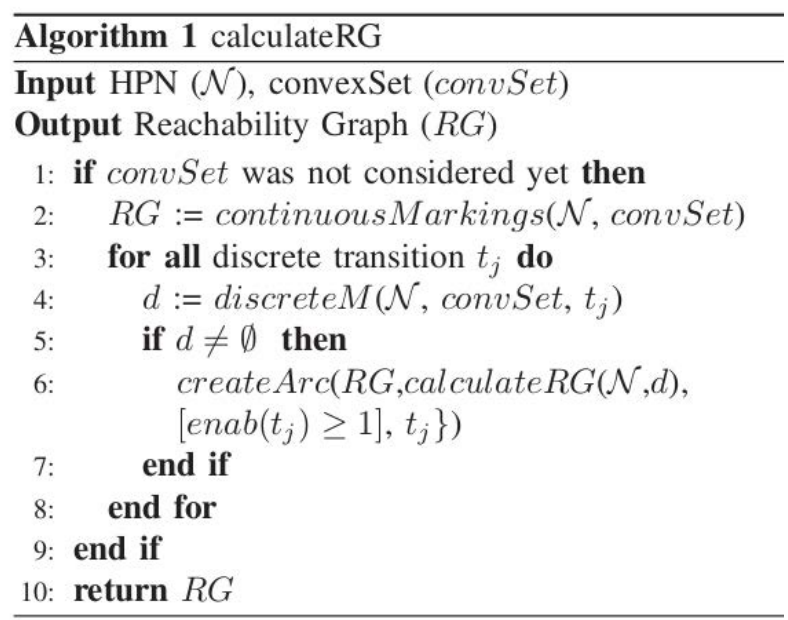

Algorithm 1 characterizes the RG. It starts in the initial marking (calculateRG $\left(\left\{\mathbf{m}_{0}\right\}\right)$ ), and it obtains the markings reachable from the initial state due to continuous and discrete firings.

In the algorithm, continuousMarkings( $N$,convSet) obtains the markings due to continuous firings from convSet, as defined for continuous PN (Júlvez et al. 2003); and discreteMarkings( $N$, convSet,tj) considers only the markings of convSet in which $t j$ is enabled, and the markings which are obtained from them. The algorithm is recursively called, 
II Reunión Jóvenes Investigadores del Instituto de Investigación en Ingeniería de Aragón (I3A)

until no new reachable regions are computed. The RS is obtained as the union of every set of the RG.

\section{Example}

Let us compare the algorithm proposed here with other techniques proposed in the literature for the computation of the RG of HPN.

The HPN depicted in Fig. 1 is taken from David and Alla (2010), in which transitions $t 1$ and $t 2$ are discrete and transitions $t 3$ and $t 4$ are continuous. The algorithm starts with the initial marking $\mathbf{m}_{0}=$ $(2,0,1,2)$ and it calculates its RG, see Fig. 2.

The RG obtained with the algorithm (Fig. 2) has three nodes, and each of them contains a convex set in $\mathbb{R}^{|P|} \geq 0$. The firings of the discrete transitions make the marking move from one convex set to another (in the case of $t 1$, respecting the guard [m[p1 ] $\geq$ 1]). Moreover, the firings of the continuous transitions are codified inside of the reachable convex sets.

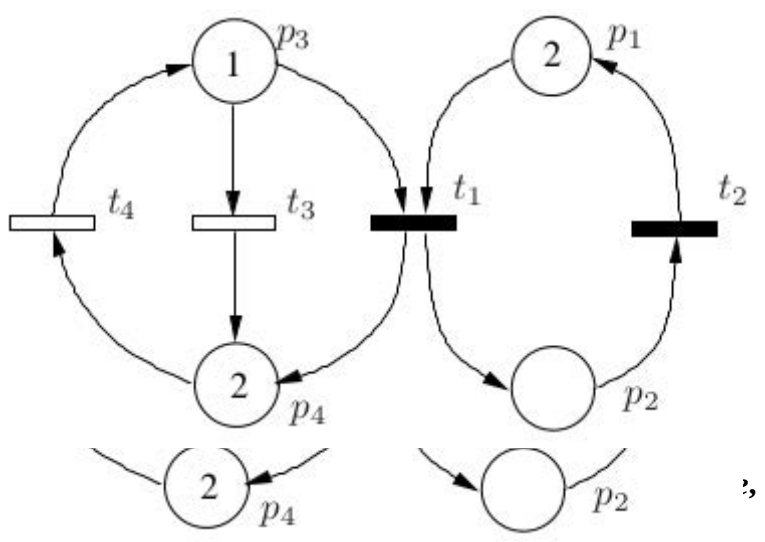

Using the technique proposed by David and Alla (2010), the RG of this HPN (Fig. 4.21, page 140) is represented with 9 nodes together with the interactions of discrete and continuous transitions among those nodes. The firing of a the discrete transition in the graph represents that it has been fired and amount of 1 . However, the firing of continuous transitions is not measured explicitly in the arcs of the RG. In our method, the firing of discrete transitions is also explicit, but the firing of continuous transitions is compacted inside the convex sets. Notice that guards for the firing of $t 1$ are needed in both methods.

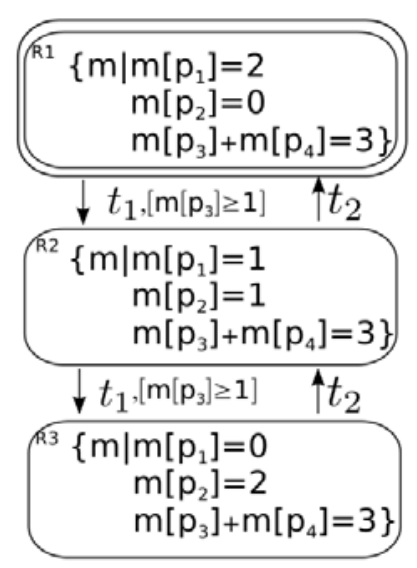

Fig. 2. Reachability Graph of the untimed HPN in Fig. 1.

\section{Conclusions}

In conclusion, the Reachability Graph of Hybrid Petri nets can be characterized as a certain number of convex sets, due to the continuous firings of transitions; connected with arcs, due to the discrete firings. Each of the convex sets is characterized with several equations and inequations. An algorithm for its computation has been proposed here, and it has being used for the computation of the Reachability Graph of a HPN system example.

\section{BIBLIOGRAPHY}

David, R. and Alla, H. Discrete, Continuous and Hybrid PetrAutonomous and timed continuous Petri nets. Springer, 2nd edition, 2010.

Júlvez, J. , Recalde, L. and Silva, M. On reachability in autonomous continuous Petri net systems. 24th International Conference on Application and Theory of Petri Nets (ICATPN 2003), Lecture Notes in Computer Science, Springer 2003.

Balduzzi, F., Di Febbraro, A., Giua, A. and Seatzu, C. Decidability Results in First-Order Hybrid Petri Nets. Discrete Event Dynamic Systems, Vol. 11, No. 1 \& 2, pp. 41-58, 2001. 